\title{
Danggui Buxue Decoction in the Treatment of Metastatic Colon Cancer: Network Pharmacology Analysis and Experimental Validation
}

This article was published in the following Dove Press journal: Drug Design, Development and Therapy

\author{
Shi-Han Feng ${ }^{l, *}$ \\ Bin Zhao ${ }^{1, *}$ \\ Xue Zhan ${ }^{2}$ \\ Retsepile Motanyane ${ }^{2}$ \\ Shu-Mei Wang ${ }^{2}$ \\ Ao $\mathrm{Li}^{3}$ \\ 'Yong Chuan Hospital of Chongqing \\ Medical University, College of Traditional \\ Chinese Medicine, Chongqing Medical \\ University, Chongqing, People's Republic \\ of China; ${ }^{2}$ College of Traditional Chinese \\ Medicine, Chongqing Medical University, \\ Chongqing Key Laboratory of Traditional \\ Chinese Medicine for Prevention and \\ Cure of Metabolic Diseases, Chongqing, \\ People's Republic of China; ${ }^{3}$ Yong Chuan \\ Hospital of Chongqing Medical University, \\ Chongqing, People's Republic of China
}

*These authors contributed equally to this work
Correspondence: Shu-Mei Wang

College of Traditional Chinese Medicine, Chongqing Medical University, No. I Medical College Road, Chongqing, 4000 16, People's Republic of China Tel +86- I87|6369329

Email wangshumei@cqmu.edu.cn

Ao Li

Yong Chuan Hospital of Chongqing

Medical University, NO. 439 Xuan Hua

Road, Yongchuan District, Chongqing,

402 I60, People's Republic of China

Tel +86-18580779649

Email wo_shi_li_ao_2@l63.com
Purpose: This study aimed to reveal Danggui Buxue Decoction (DBD) candidate targets and mechanisms in the treatment of metastatic colon cancer (MCC), using network pharmacology-based analyses and experimental validation.

Methods: Traditional Chinese Medicine Systems Pharmacology (TCMSP) database query and text mining were used to screen active compounds in DBD, and the Swiss target prediction platform was applied to predict compound-related target proteins. Targets likely associated with MCC were determined using GeneCards and OMIM databases. Targets common to DBD and MCC were obtained from the Venn platform; subsequently, Cytoscape was used to construct drug-compound-target-disease and protein-protein interaction networks. The hub gene was determined by R, while GO and KEGG enrichment analyses were performed on common targets to elucidate biological processes and signaling pathways involved in DBD against MCC. Finally, the metastatic colon cancer mouse model was used to detect the levels of expression of protein Bax, Bcl2, Caspase3, and Cleaved caspase 3 by Western blot.

Results: A total of 28 active compounds and 61 common targets were predicted. The main compounds were quercetin, hederagenin, jaranol, methylnissolin, formononetin, calycosin, kaempferol, 3.9-di-O-methylnissolin, 24-propylcholesterol, and 7-O-methylisomucronulatol, present in Astragalus membranaceus (Huangqi, HQ). In addition, beta-sitosterol, ferulic acid, and stigmasterol, present in Angelica sinensis (Danggui, DG), were detected. JUN, PTSG2, EGFR, ESR1and, CASP3 genes were the top 5 hub genes in the PPI network. GO and KEGG enrichment analyses indicated that apoptosis played a major role in the biological processes and signaling pathways involved. Moreover, the in vivo experiment revealed that DBD inhibited MCC by up-regulating the expression of Bax, Caspase3, and Cleaved caspase3, and by down-regulating the expression of $\mathrm{Bcl} 2$.

Conclusion: This study revealed candidate DBD targets and mechanisms in the treatment of MCC, using network pharmacology-based analyses and experimental validation. The present findings provide a reference for tumor treatment during the perioperative period.

Keywords: Danggui Buxue Decoction, network pharmacology, primary tumor, metastatic tumor, perioperative period

\section{Introduction}

Colon cancer is the third most frequently diagnosed malignant tumor and the second leading cause of cancer-related deaths worldwide. ${ }^{1}$ Surgery as the primary treatment for malignant tumors has significantly improved the prognosis and quality of life of cancer patients. The risk of metastatic tumor development 
following surgery remains the major factor associated with the limited efficacy of the operation. Evidence suggests that the perioperative period and excision of the primary tumor can promote the development of metastases, which is the main cause of cancer-related mortality. ${ }^{2-4}$ The perioperative period of approximately 5-7 days before and 7-12 days after tumor excision is pivotal in determining long-term cancer outcomes and the risk of postoperative metastatic disease. Although surgical removal of the primary tumor can reduce the tumor burden, it can also facilitate the development of metastases through numerous mechanisms, for example, cell immunosuppression and stress response, ${ }^{5-7}$ tumor angiogenic switch imbalance, ${ }^{8,9}$ tumor cell dissemination through the blood and lymphatic systems, and the release of growth factors. ${ }^{10,11}$ These processes work synergistically during the perioperative period, significantly increasing the risk of metastases. This evidence suggests that the perioperative period may be used to suppress metastatic progression and improve oncological outcomes. However, conventional chemoradiotherapy cannot be used during this period, given its suppressive effects on the immune system and tissue healing. ${ }^{4}$ Thus, there is a need for an alternative approach.

Reinforcing qi and nourishing the blood are among the approaches used in conventional traditional Chinese medicine (TCM) to correct blood loss resulting from operation. Danggui Buxue Decoction (DBD) is a classic Chinese prescription first described in "NeiwaishangBianhuoLun" written by Dong-yuan Li in China (Jin Dynasty, CE 1247). ${ }^{12}$ It includes the root of Astragalus membranaceus (Fisch.) Bunge. (Huangqi, HQ) and the root of Angelica sinensis (Oliv.) Diels. (Danggui, DG) at the ratio of 5:1 and is used for treating qi and the blood deficiency syndrome. ${ }^{13}$ Studies have shown that DBD can attenuate apoptosis of hematopoietic stem cells in the mouse model of aplastic anemia, ${ }^{14}$ improve bone marrow suppression induced by cyclophosphamide by promoting the synthesis and secretion of hematopoietic growth factors and the proliferation of hematopoietic progenitor cells, ${ }^{15}$ and promote dermal wound healing by reducing the level of inflammatory response. ${ }^{16}$ In addition, DBD can promote immune response by increasing the levels of lymphocytes, macrophages, and interleukin-2. ${ }^{17}$ This decoction is widely used in clinical practice due to its therapeutic effects. Recent pharmacological studies have shown the evidence of anti-tumor effects of DBD; in fact, the use of DBD for NSCLC patients at an early postoperational stage has been associated with alleviated impairment and accelerated recovery of patients' immune function. ${ }^{18}$ Different concentrations of DBD may inhibit the proliferation of endothelial cells co-cultured with A549 lung cancer cells. ${ }^{19}$ Moreover, DBD has been shown to significantly inhibit the formation of lung metastases in the Lewis lung cancer mouse model. However, little is known about the molecular mechanisms of DBD against MCC.

Network pharmacology uses systems biology theory to predict potential mechanism of drug action in the treatment of diseases through compound-target-disease interaction network and bioinformatics analysis, ${ }^{20}$ which are consistent with the holistic perspective of TCM.

This study aimed to explore the anti-tumor effects of DBD on MCC during the perioperative period. Network pharmacology and bioinformatics analyses were used to predict candidate compounds and mechanisms of DBD in the treatment of MCC. In addition, an in vivo experiment was conducted to detect the levels of expression of apoptosis-related protein $\mathrm{Bcl} 2$, Bax, Caspase3 (Cas3), and Cleaved caspase3 (C-cas3) to provide a reference for the application of tonic therapy of tumor treatment in the perioperative period.

\section{Materials and Methods Drug, Reagents and Cell Line}

A. membranaceus (Inner Mongolia Autonomous Region, China, batch number 191,210,600) and A. sinensis (Gansu province, China, batch number 200,501) were purchased from the First Affiliated Hospital of Chongqing Medical University (Chongqing, China). Primary antibodies against $\mathrm{Bcl} 2$ (AF6139), Bax (AF0120), C-cas3 (AF7022) and $\beta$ actin (AF7018) were purchased from Affinity (Cincinnati, USA). Cas3 (27,525); and secondary antibodies goat anti-rabbit Ig $\mathrm{G}$ and goat anti-mouse $\lg \mathrm{G}$ were purchased from Signalway Antibody (College Park, Maryland, USA). Mouse colon cancer cell line CT26 (CL-0071) was purchased from Procell Life Science\&Technology Co., Ltd (Wuhan City, China) and cultured in RPMI 1640 (BI, Kibbutz Beit Haemek, Israel), supplemented with $10 \%$ fetal bovine serum (Lot1922693, BI, Kibbutz Beit Haemek, Israel) and $1 \%$ penicillin/streptomycin (Beyotime, shanghai, China). All cultures were maintained in a humidified environment with $5 \% \mathrm{CO}_{2}$ at $37^{\circ} \mathrm{C}$. 


\section{Network Pharmacology Analyses}

Active Compounds and Protein Targets Identification of DBD

Data on DBD active compounds were collected from the TCMSP database (http://lsp.nwsuaf.edu.cn), using the ADME filter method whose main parameters included oral bioavailability (OB) and drug-likeness (DL). OB of $\geq 30 \%$ and $\mathrm{DL}$ of $\geq 0.18$ were the threshold values for ADME evaluation systems. Compounds such as astragalus polysaccharide, astragaloside, astragaloside III, astragaloside IV, alpha-Angelica lactone and ferulic acid, which exhibit antitumor effect, were identified as potential compounds in DBD via text mining. The CAS number of each compound was uploaded to the Pubchem platform (https://pubchem. ncbi.nlm.nih.gov/) to obtain its 2D structure; subsequently, the Swiss Target Prediction platform (http://www.swisstar getprediction.ch/) and TCMSP database were used to identify compounds-related targets; at this stage, zero probability and repeated targets were removed. The human gene symbol corresponding to the protein target name was standardized using the Uniprot database (https://www.uniprot.org/).

\section{MCC-Related Targets Prediction}

Target genes associated with MCC were acquired from GeneCards (https://www.genecards.org/) and OMIM (https://omim.org/) databases. The targets common in DBD and MCC were identified by the Venn platform (http://bioinformatics.psb.ugent.be/webtools/Venn/) and included in further analysis.

\section{Network Construction}

Data on the compounds and common targets were imported into Cytoscape 3.7.2 to construct the drug-compound-target -disease interaction network. The topological parameters of each node, including the degree centrality (DC), betweenness centrality (BC) and closeness of centrality (CC) were evaluated by NetworkAnalyzer plug-in for Cytoscape. String 11.0 (https://string-db.org/) was used to determine protein-protein interactions, given the human species, medium confidence score of 0.4 , and interaction data derived from literature mining, experiments, and databases; finally, the TSV document of string-interactions was visualized by Cytoscape.

\section{GO and KEGG Pathway Enrichment Analysis}

Cluster analysis of biological process of GO was performed with the CluGo2.5.7 plug-in for Cytoscape. To obtain the potential signaling pathway associated with DBD in the treatment of MCC, KEGG analysis was conducted by $\mathrm{R}$ 4.0.0 with the Bioconductor program packages clusterProfiler 3.16.1 and dose 3.14.0 installed.

\section{Preparation of DBD}

HQ and DG were mixed in the ratio of 5:1 (dry weight) and immersed in 8 volumes of distilled water $(\mathrm{v} / \mathrm{m})$ for 0.5 $\mathrm{h}$, then decocted twice, each time for $1.5 \mathrm{~h}$. This ratio followed the ancient recipe that has been shown to have the best extracting efficiency. ${ }^{21,22}$ Subsequently, the waterbased decoction was combined, filtered, and concentrated to $0.6 \mathrm{~g} / \mathrm{mL}$, and stored at $-80{ }^{\circ} \mathrm{C}$ ahead of the experiment.

\section{Animals and Experimental Design}

All protocols involving animals were approved by the Ethics Committee of Yongchuan Hospital affiliated with the Chongqing Medical University (NO: YCHCQMU2017022521), and the guidelines followed for the welfare of the laboratory animals was GB/T 35,892-2018 Laboratory Animal Guidelines for Ethical Review of Animal Welfare. Sixty male BABL/c mice aged 5-6 weeks (weighing 16 $\pm 2 \mathrm{~g}$ ) were purchased from the Laboratory Animal Center of the Chongqing Medical University (SCXK-(yu)2018-0003). All mice were housed in standard husbandry conditions $\left(22 \pm 2^{\circ} \mathrm{C}, 60-80 \%\right.$ relative humidity, and a 12-hour photoperiod) and given free access to food and water during the course of the experiment. After 1 week of adaptation, 200 ul $5 * 10^{6}$ CT26 single cell suspension was injected subcutaneously into the right axilla as primary tumor. Five days later, equal volume and density CT26 suspension was injected into the opposite axilla as metastatic tumor. ${ }^{23-25}$ The mice were then randomly divided into 4 groups $(n=15$ per group), primary tumor resected group (R), non-resected primary tumor group (NR), and DBD I group, and DBD II group. Primary tumor resection surgery was performed in all groups except the NR group. Group DBD I received $2 \mathrm{~mL} / 100 \mathrm{~g}$ of DBD 7 days before surgery; meanwhile, treatment in groups $\mathrm{R}(2 \mathrm{~mL} / 100 \mathrm{~g}$, distilled water), NR ( $2 \mathrm{~mL} / 100 \mathrm{~g}$, distilled water), and DBD II $(2 \mathrm{~mL} / 100 \mathrm{~g}$, DBD) started 24 hours after the operation. All mice were treated for 14 consecutive days. Tumor volume was measured with vernier caliper every 3 days and calculated using the formula: Volume $\left(\mathrm{mm}^{3}\right)=1 / 2 \mathrm{AB}^{2}$, where $A$ and $B$ represented the length and width values, respectively. Animals were sacrificed after the 14th intragastric administration; each tumor mass was removed and 
weighed. Subsequently, tumor tissue specimens were fixed in $4 \%$ paraformaldehyde before embedding them in paraffin; then, sections of $5 \mu \mathrm{m}$ thickness were cut and stained with hematoxylin and eosin. Histopathological changes in the tumor tissues of groups R, NR, DBD I, and DBD II were evaluated using a light microscope.

\section{Western Blot Assay}

The total protein of metastatic tumor was extracted with RIPA lysis buffer (Beyotime, shanghai, China) containing 1\% PMSF. Protein concentration was determined by BCA protein assay reagent (Beyotime, shanghai, China), according to the manufacturer's instructions. Proteins were separated by SDS-PAGE gels and transferred onto PVDF membranes (Millipore, USA). After blocking with 5\% nonfat milk, the membranes were incubated with primary antibodies $\operatorname{Bcl} 2$ (1:1000), Bax (1:500), Cas3 (1:1000), and C-cas3 (1:500) at $4{ }^{\circ} \mathrm{C}$ overnight; in addition, $\beta$-actin (1:5000) was used as a housekeeping gene. Subsequently, the membranes were washed and incubated for 2 hours at room temperature with HRP-conjugated secondary antibodies. Protein bands were detected by ECL Western Blotting Detection Kit (4A Biotech, Beijing, China) and quantified by Image J software. Each experiment was repeated three times.

\section{Statistical Analysis}

Statistical analysis was performed by SPSS Statistics 25 (IBM Corporation, Armonk, NY, USA) using independent-samples $t$ test. Results were expressed as the mean \pm standard deviation. P-values of $<0.05$ were considered indicative of a statistically significant.

\section{Results}

\section{Active Compounds and Related Targets Information of DBD}

A total of 22 DBD compounds were retrieved from the TCMSP database, using the screening criteria. Compounds such as astragalus polysaccharide, astragaloside, astragaloside III, astragaloside IV, alpha-angelica lactone, and ferulic acid were recognized as active compounds and included in further analysis (Table 1). A total of 596 and 132 targets were predicted in HQ and DG, respectively.

\section{MCCTargets Prediction and the Common Targets}

A total of 6800 targets related to MCC were obtained from the GeneCards and OMIM databases. A higher relevance score indicated a higher correlation between a gene and MCC; a total of 1751 targets with the relevance score higher than twice the median value were considered candidate therapeutic targets. After comparing the targets of DBD with those of MCC, 61 matched targets were identified (Supplementary Table S1), and a Venn diagram was plotted (Figure 1). The common targets were regarded as the potential therapeutic targets for DBD against MCC.

\section{Network Visualization}

To clarify the relationship between active compounds and common targets as well as MCC, a drug-compound-targetdisease interaction network with 90 nodes and 458 edges was created by Cytoscape (Figure 2). It contained 26 active compounds and 61 potential targets; at this stage, astragaloside III and alpha-angelica lactone were excluded due to the absence in them of common targets against MCC. The average DC, BC, and CC values were 16.15 , 0.01 , and 0.42 , respectively, for the compounds. There were 13 compounds with DC values higher than the average value, namely, quercetin, hederagenin, jaranol, methylnissolin, formononetin, calycosin, kaempferol, 3.9-di-O-methylnissolin, 24-propylcholesterol, and 7-O-methylisomucronulatol, which belonged to $\mathrm{HQ}$, and beta-sitosterol, ferulic acid, and stigmasterol, which belonged to DG (Table 2). These compounds were considered the main active components of DBD in the treatment of MCC with a high degree value. String platform was employed to explore the interaction among common targets; subsequently, a PPI network was constructed by Cytoscape (Figure 3A). This network comprised 59 nodes (AKR1B10, NQ02 were concealed as disconnected nodes in the network) and 404 edges, with the average degree value of 13.69; $\mathrm{R}$ was used to hunt hub genes (Figure 3B). The greater connectivity between the nodes, the more important the nodes in this network. JUN, PTSG2, EGFR, ESR1, and CASP3 were the top five target genes in the PPI network and the main targets of DBD (Table 3).

\section{GO and KEGG Enrichment Analysis}

To explore the biological processes of GO regulated by DBD, cluster analysis of common targets was performed through CluGo for the human species, $P \leq 0.05$, and the Kappa score threshold value of 0.4 . A total of 42 biological processes were enriched (Figure 4A) and formed 15 clusters (Figure 4B). Terms with the same color belonged to the same cluster: nuclear receptor activity, steroid hormone receptor activity, myeloid leukocyte differentiation, 


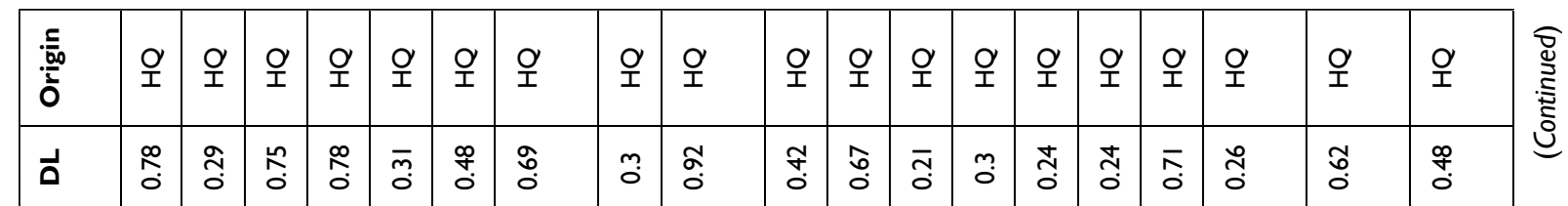

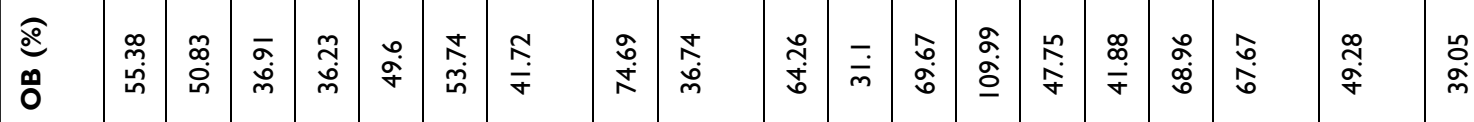

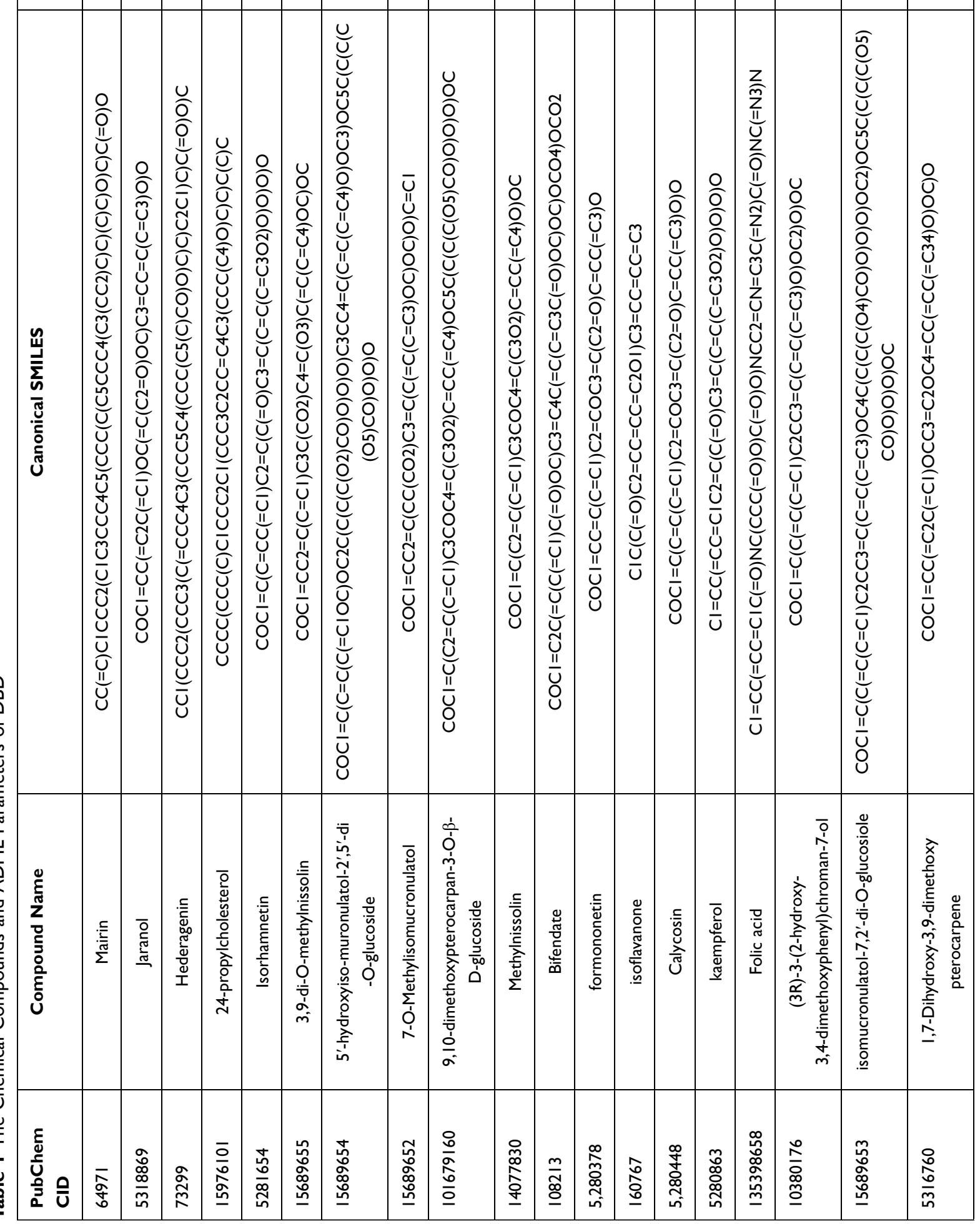




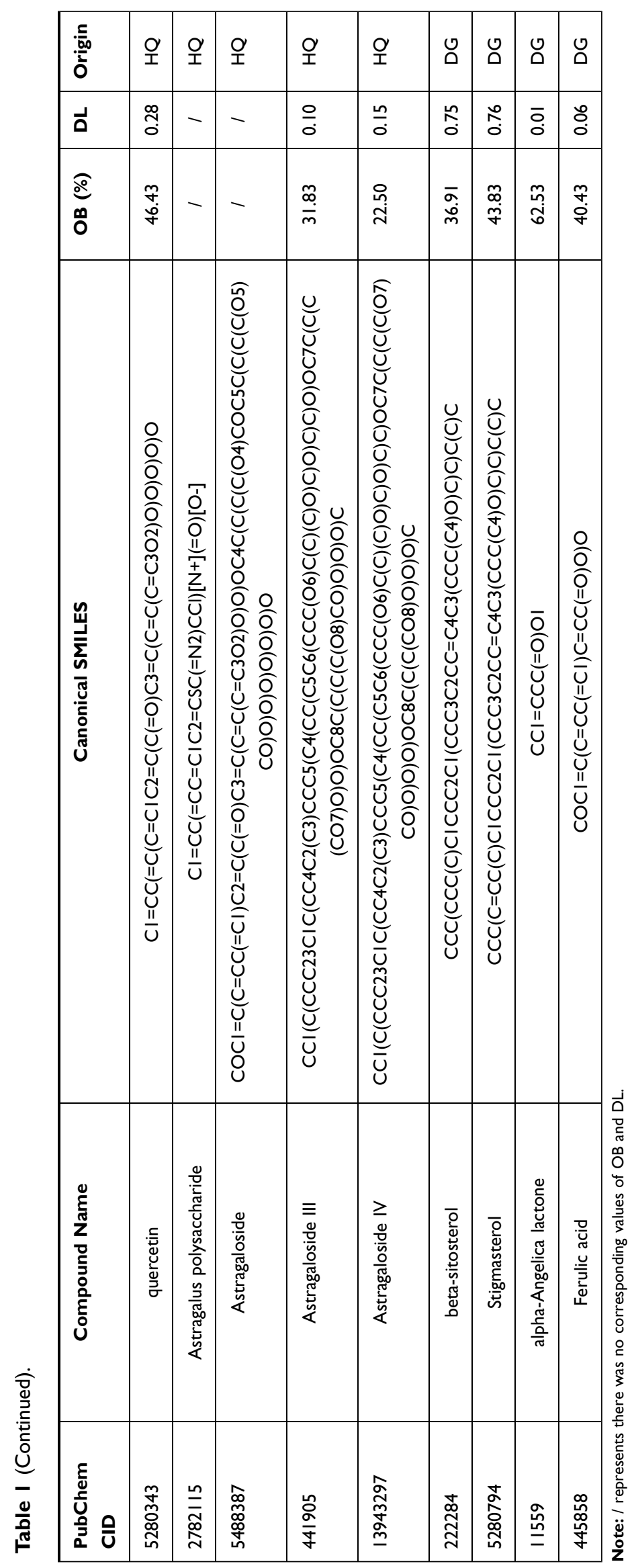




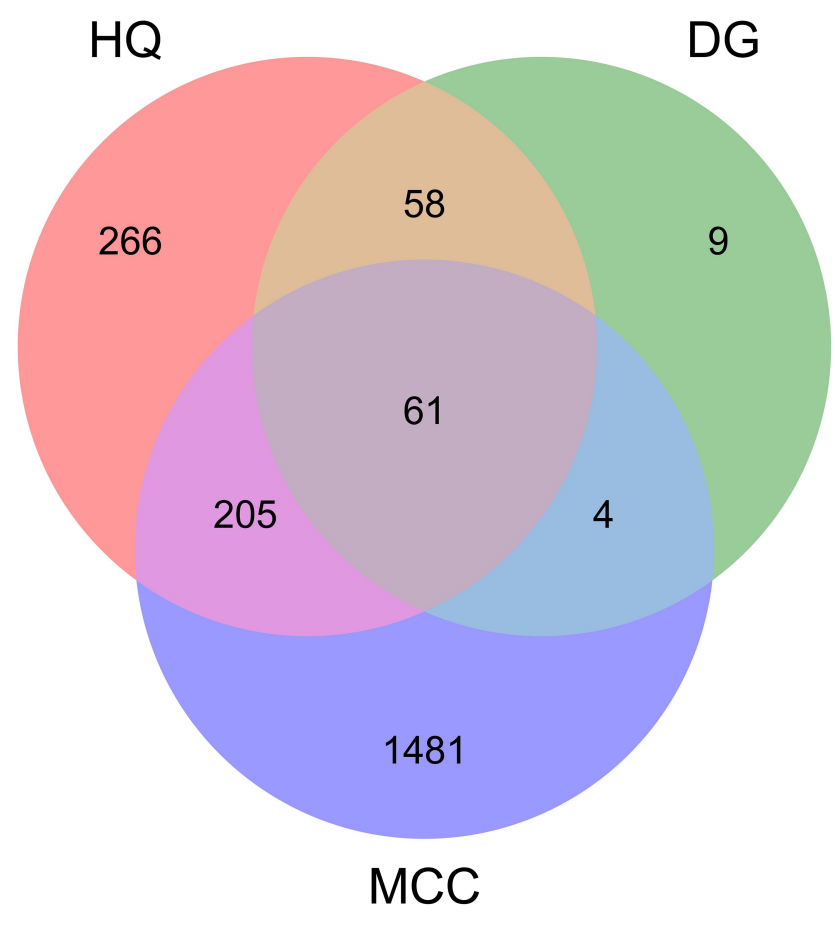

Figure I Venn diagram of common targets in DBD and MCC.

positive regulation of bone resorption, icosanoid metabolic process, regulation of intrinsic apoptotic signaling pathway, positive regulation of smooth muscle cell proliferation, placenta development, positive regulation of neuron death, response to molecules of bacterial origin, cell death in response to oxidative stress, diterpenoid metabolic process, activation of cysteine-type endopeptidase activity involved in the apoptotic process, positive regulation of NIK/NF-kappa B signaling, and regulation of cardiac muscle hypertrophy were the leading terms, based on the highest significance value in each cluster. The KEGG pathway enrichment analysis was performed by $\mathrm{R}$ with FDR of $\leq 0.05$ (Figure 5). Among the top 30 signaling pathways, Apoptosis-multiple species (hsa04215), Apoptosis (hsa04210) and Colorectal cancer (hsa05210) were involved in regulating $\mathrm{MCC}$ progression. These findings suggest that DBD likely inhibited MCC by regulating the biological processes and pathways related to apoptosis; the subsequent in vivo experiment helped verify this hypothesis.

\section{DBD Alleviated MCC Progression in Mice}

Palpable tumors were observed on day 5 after tumor cell inoculation. The tumor growth rate decreased successively in groups R, NR, DBD I, and DBD II (Figure 6A). After 2 weeks of treatment with postoperative administration of
DBD, reduction in tumor weight of $74.3 \%$ was observed in the DBD II group, compared to the R group ( $p<0.0001$ ), and non-excision of primary tumor caused a reduction in tumor weight of $25.1 \%$ compared with the $\mathrm{R}$ group $(p<0.001)$. The mean values of tumor weight were $2.41 \pm$ $0.30 \mathrm{~g}, 1.81 \pm 0.17 \mathrm{~g}, 1.34 \pm 0.15 \mathrm{~g}$, and $0.62 \pm 0.13 \mathrm{~g}$ for groups R, NR, DBD I, and DBD II respectively (Figure 6B and Supplementary Figure S1). Neoplastic cells exhibited increased nucleo-cytoplasmic ratio, nuclear atypia rate, and hyperchromatic nuclei count in group $\mathrm{R}$. Treatment with DBD reduced the nucleo-cytoplasmic ratio as well as the volume of neoplastic cells, increasing the rate of necrosis (Figure 7). These findings indicate that DBD may inhibit MCC progression.

\section{Expression of Bax, Bcl2, Cas3, and C-Cas3 in Metastatic Tumor}

Western blot analysis indicated that the expression of Bax, Cas3, and C-cas3 was up-regulated in the DBD I/II groups compared to that in the R group; moreover, the expression of these proteins in the DBD II group was markedly upregulated compared to that in the $\mathrm{R}$ group $(p<0.001$, $p<0.01)$. Meanwhile, it was down-regulated in the $\mathrm{R}$ group compared to the NR group $(p<0.05, p<0.01)$ (Figure 8$)$. In contrast, the expression of $\mathrm{Bcl} 2$ was down-regulated in the DBD I/II groups compared to that in the R group; moreover, in the DBD II group, it was significantly down-regulated compared to that in the $\mathrm{R}$ group $(p<0.0001)$. Finally, the expression of $\mathrm{Bcl} 2$ in the $\mathrm{R}$ group was up-regulated compared to that in the NR group $(p<0.01)$ (Figure $8 \mathrm{C})$. In addition, we calculated the ratio of $\mathrm{Bax} / \mathrm{Bcl} 2$ in four groups; in the DBD I/II groups, it increased more than it did in the $\mathrm{R}$ group, while in the $\mathrm{R}$ group, it was lower than in the $\mathrm{DBD}$ II and NR groups (Figure 8D). These findings indicate that DBD may induce metastatic tumor apoptosis by upregulating Bax, Cas3, and $\mathrm{C}$-cas3, and down-regulating $\mathrm{Bcl} 2$ protein expression.

\section{Discussion}

Clinical practice and many mice models have proven that surgical removal of the primary tumor facilitates the development of metastases. ${ }^{5,8}$ We hypothesize that DBD could inhibit metastatic colon cancer progress after the primary tumor removal. In the present study, the candidate DBD compounds and targets against MCC were predicted using network pharmacology, which yielded 28 compounds and 61 targets. The present findings 


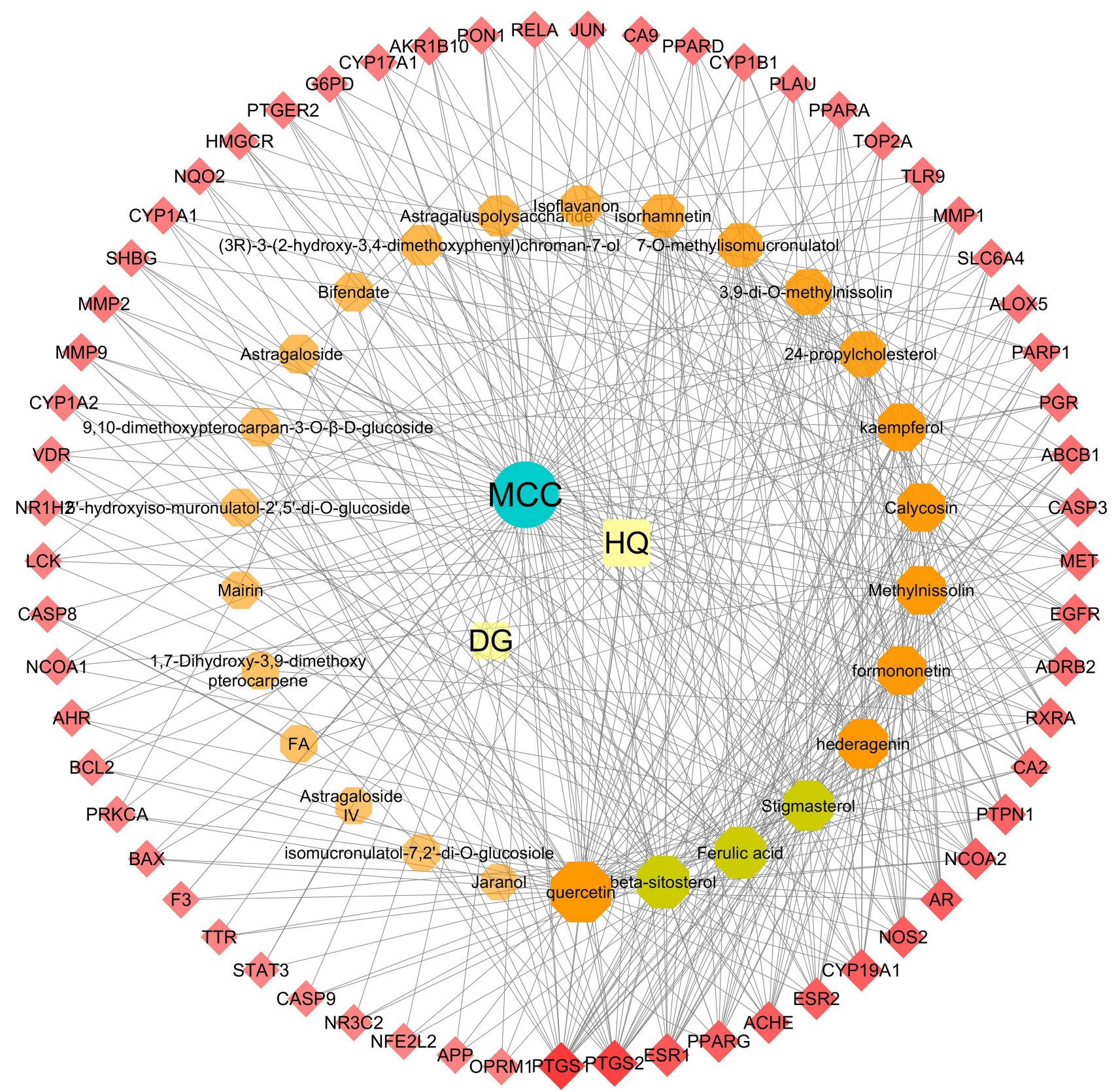

Figure 2 DBD-compound-target-MCC network. All nodes were visualized in degree value, the larger the node, the darker the color and the higher the degree value. In this network, the red diamonds represent candidate targets of DBD against MCC, the orange and claybank octagons represent active compounds in $\mathrm{HQ}$ and DG, the green ellipse represent disease, while the remaining golden squares represent HQ and DG.

indicated that the main DBD compounds involved in the treatment of MCC were quercetin, hederagenin, jaranol, methylnissolin, formononetin, calycosin, kaempferol, 3.9-di-O-methylnissolin, 24-propylcholesterol, 7-O-methylisomucronulatol, beta-sitosterol, ferulic acid, and stigmasterol. Previous pharmacological research has shown that a combination of quercetin and 2-methoxyestradiol enhanced the inhibition of human prostate cancer LNCaP and PC-3 cells xenograft tumor growth. ${ }^{26}$ A previous in vitro experiment indicated that hederagenin induced apoptosis in breast cancer cells by regulating the mitochondrial apoptosis pathway. $^{27}$ Kumatakenin (jaranol) induced caspase-dependent apoptosis in human ovarian cancer cells by increasing the expression of caspase- $3,-8$, and $-9 .{ }^{28}$ Meanwhile, formononetin inhibited the proliferation and metastasis of ovarian cancer in vitro; ${ }^{29}$ finally, calycosin induced apoptosis of cervical cancer and adenocarcinoma. ${ }^{30,31}$ 
Table 2 The Topological Parameter Analysis of Top 13 Compounds in DBD

\begin{tabular}{|l|c|c|c|c|}
\hline Number & Compound & Degree Centrality & Betweenness Centrality & Closeness Centrality \\
\hline 1 & Quercetin & 49 & 0.12 & 0.57 \\
\hline 2 & Beta-sitosterol & 33 & 0.06 & 0.49 \\
\hline 3 & Ferulic acid & 31 & 0.06 & 0.49 \\
\hline 4 & Stigmasterol & 28 & 0.04 & 0.47 \\
\hline 5 & Hederagenin & 0.03 & 0.46 \\
\hline 6 & Jaranol & 27 & 0.03 & 0.46 \\
\hline 7 & Methylnissolin & 26 & 0.03 & 0.46 \\
\hline 8 & Formononetin & 25 & 0.02 & 0.45 \\
\hline 9 & Calycosin & 24 & 0.02 & 0.45 \\
\hline 10 & Kaempferol & 23 & 0.03 & 0.45 \\
\hline 11 & $3,9-$ di-O-methylnissolin & 19 & 0.02 & 0.43 \\
\hline 12 & $24-$ propylcholesterol & 19 & 0.02 & 0.43 \\
\hline 13 & $7-0$-methylisomucronulatol & 17 & 0.01 & \\
\hline
\end{tabular}

A

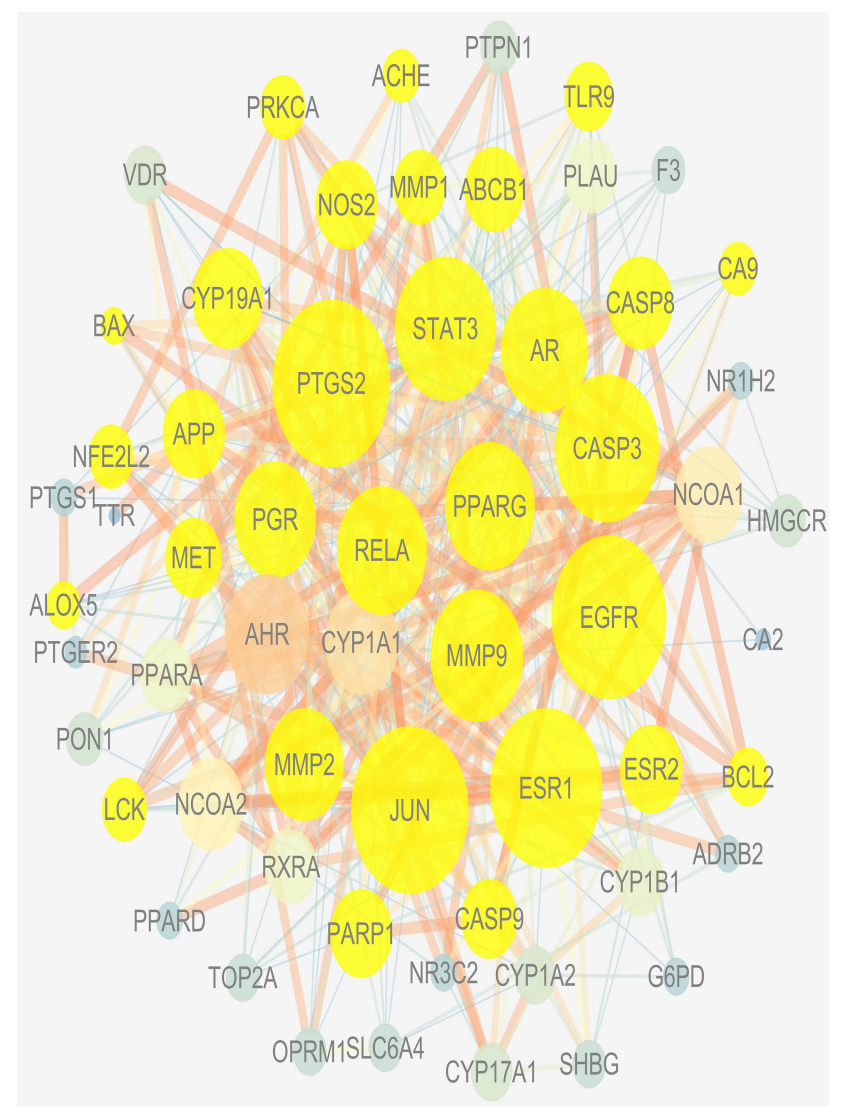

B

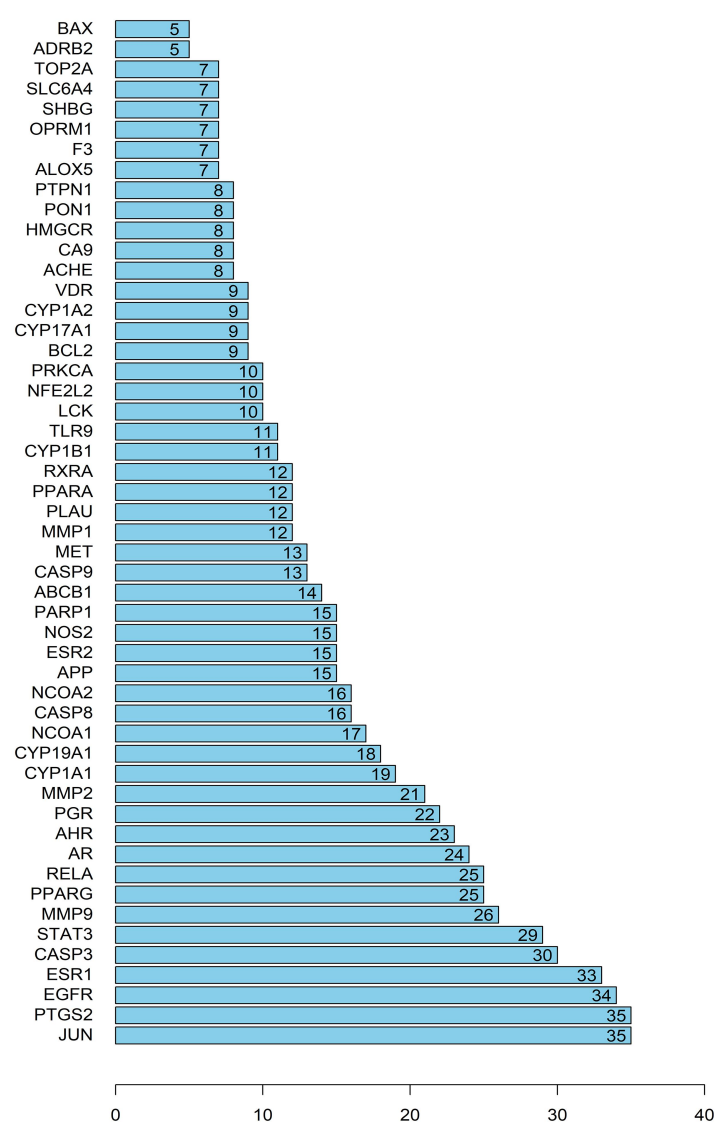

Figure 3 The construction of protein-protein interaction network. (A) Protein-Protein interaction network. Edges represent protein-protein interactions, edge thickness indicates the strength of data support, the larger the node, the higher the degree value. Golden circles represent the targets that interact with CASP3. (B) Common Targetsbar plot diagram. $\mathrm{X}$-axis represents the number of nodes connected and Y-axis represents the target gene symbol. 
Table 3 Parameters of the Top 30 Genes in the PPI Network

\begin{tabular}{|c|c|c|c|c|c|}
\hline Number & Gene & Degree & Betweenness Centrality & Closeness Centrality & Connected Nodes \\
\hline I & PTGS2 & 35 & 0.10 & 0.72 & 35 \\
\hline 2 & JUN & 35 & 0.06 & 0.72 & 35 \\
\hline 3 & EGFR & 34 & 0.08 & 0.71 & 34 \\
\hline 4 & ESRI & 33 & 0.07 & 0.70 & 33 \\
\hline 5 & CASP3 & 30 & 0.05 & 0.67 & 30 \\
\hline 6 & STAT3 & 29 & 0.04 & 0.66 & 29 \\
\hline 7 & MMP9 & 26 & 0.05 & 0.64 & 26 \\
\hline 8 & RELA & 25 & 0.03 & 0.63 & 25 \\
\hline 9 & PPARG & 25 & 0.05 & 0.64 & 25 \\
\hline 10 & AR & 24 & 0.05 & 0.62 & 24 \\
\hline II & AHR & 23 & 0.02 & 0.62 & 23 \\
\hline 12 & PGR & 22 & 0.03 & 0.61 & 22 \\
\hline 13 & MMP2 & 21 & 0.01 & 0.60 & 21 \\
\hline 14 & CYPIAI & 19 & 0.03 & 0.59 & 19 \\
\hline 15 & CYPI9AI & 18 & 0.02 & 0.59 & 18 \\
\hline 16 & NCOAI & 17 & 0.02 & 0.57 & 17 \\
\hline 17 & CASP8 & 16 & 0.01 & 0.57 & 16 \\
\hline 18 & $\mathrm{NCOA} 2$ & 16 & 0.02 & 0.56 & 16 \\
\hline 19 & NOS2 & 15 & 0.02 & 0.55 & 15 \\
\hline 20 & APP & 15 & 0.05 & 0.57 & 15 \\
\hline 21 & PARPI & 15 & 0.01 & 0.55 & 15 \\
\hline 22 & ESR2 & 15 & 0.01 & 0.56 & 15 \\
\hline 23 & $A B C B I$ & 14 & 0.02 & 0.55 & 14 \\
\hline 24 & MET & 13 & 0.00 & 0.54 & 13 \\
\hline 25 & CASP9 & 13 & 0.00 & 0.54 & 13 \\
\hline 26 & PLAU & 12 & 0.01 & 0.54 & 12 \\
\hline 27 & MMPI & 12 & 0.00 & 0.55 & 12 \\
\hline 28 & PPARA & 12 & 0.01 & 0.53 & 12 \\
\hline 29 & $R \times R A$ & 12 & 0.01 & 0.51 & 12 \\
\hline 30 & TLR9 & 11 & 0.00 & 0.52 & 11 \\
\hline
\end{tabular}

In addition, a separate study has shown that beta- ferulic acid inhibited the proliferation and migration of sitosterol and gemcitabine exhibit synergistic anti- human lung cancer cells in vitro. ${ }^{32}$ These compounds pancreatic cancer activity by modulating apoptosis and have been shown to exert pro-apoptosis activity in many inhibiting epithelial-mesenchymal transition, while cancer cell types. Presently, the PPI network showed 


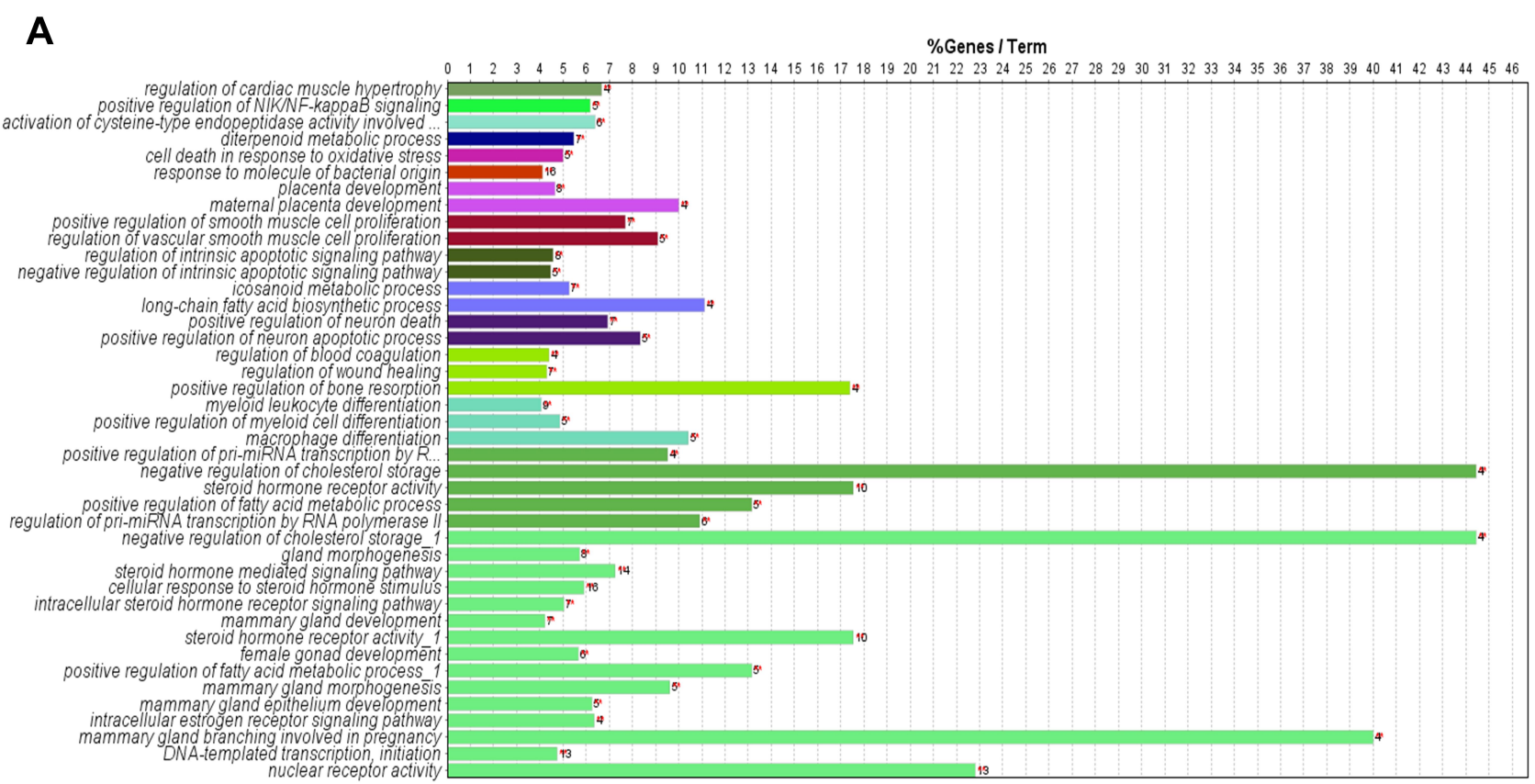

B

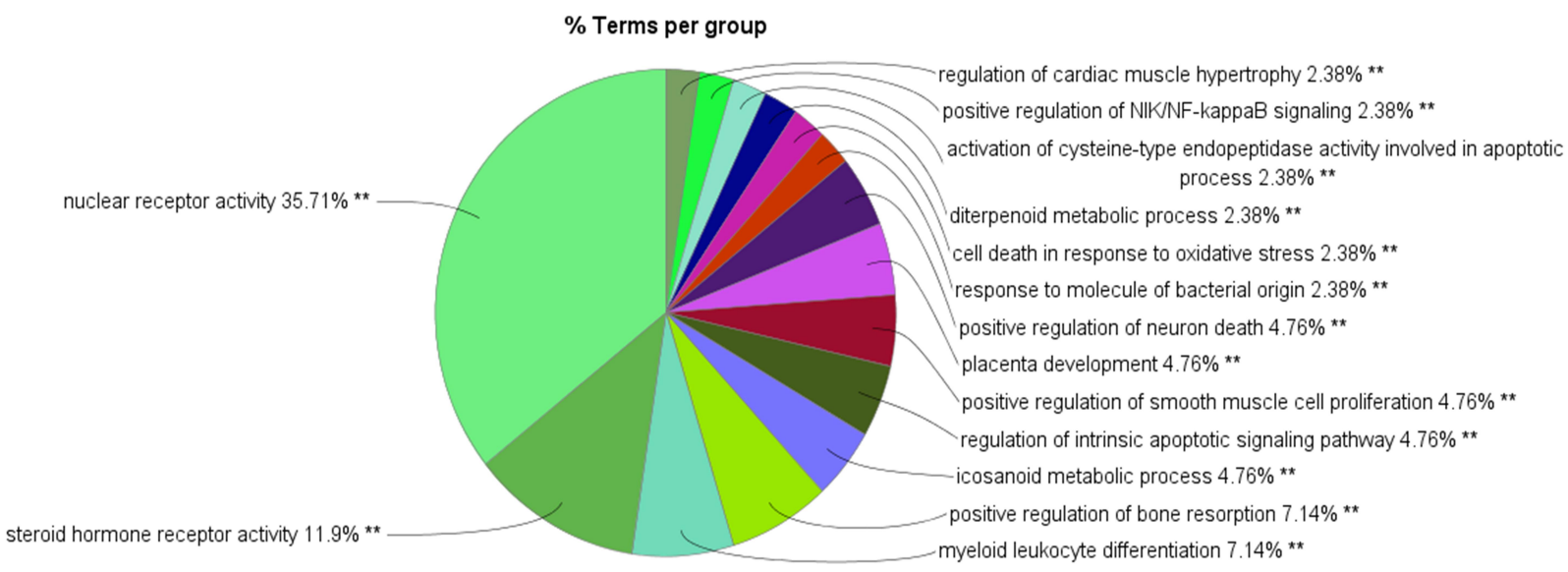

Figure $4 \mathrm{GO}$ enrichment analysis. Different colors represent different clusters. (A) Terms of enriched biological processes on common targets. X-axis represents the percentage of the target genes versus the background genes in each term, Y-axis represents the name of biological processes, the red **Represents the number of enriched target genes. (B) Percentage of each cluster. The name of each term represents the leading term based on the highest significance value in each cluster.

that Cas 3 was among the hub genes linked to 30 targets with a high degree value, closely associated with apoptosis. Findings from the GO and KEGG enrichment analyses indicated that apoptosis played a major role in the biological processes and signaling pathways of MCC.

Inadequacy and induction of apoptosis are among the hallmarks of cancer-associated and anti-tumor mechanisms, respectively. ${ }^{33}$ The process of intrinsic/mitochondrial apoptosis involves two protein families, among which the Bcl2 family regulates the mitochondrial membrane permeability, while the caspase family is the "executor" of apoptosis. The apoptotic trigger conveys signals between the "regulators" and "executors" in a way that is controlled by counter-balancing the expression of pro- and antiapoptotic members of the $\mathrm{Bcl} 2$ family of regulatory proteins. ${ }^{34}$ Anti-apoptotic protein $\mathrm{Bcl} 2$ embedded in the mitochondrial outer membrane inhibits cytochrome $\mathrm{C}$ release; meanwhile, pro-apoptotic protein Bax belonging to Bcl2 family and embedded in the cytoplasm is transported into the mitochondria after receiving cell death-related signals and promotes the release of 


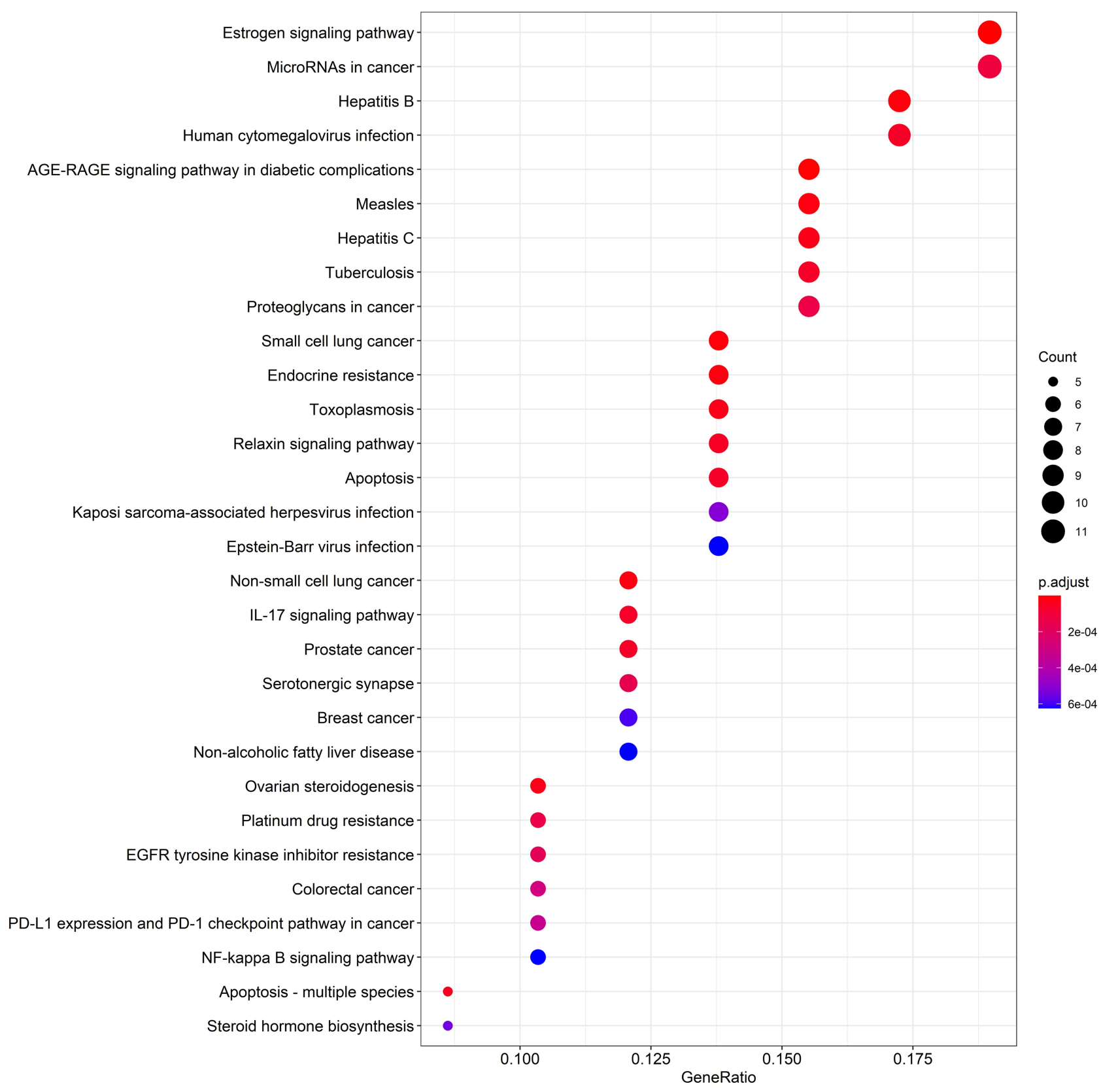

Figure 5 KEGG pathway enrichment dot-plot diagram. The top 30 signaling pathways regulated by DBD. X-axis represents the ratio of enriched target genes/background genes. Y-axis represents the term of enriched pathways. The sizes of the dots indicate the number of target genes in a certain pathway, and the colors of the dots reflect the different values of p.adjust.

cytochrome $\mathrm{C}$, which activates caspase cascade responsible for apoptosis execution. The up-regulation of Bcl2 expression as a barrier to apoptosis has been described in several types of solid tumors. ${ }^{35}$ An in vitro experiment revealed that MiR-136 triggers apoptosis in human gastric cancer cells by down-regulating $\mathrm{Bcl} 2$ expression. ${ }^{36} \mathrm{Bcl} 2-$ inhibitor venetoclax prolonged progression free survival and increased sensitivity to chemoradiotherapy in patients with ER- and Bcl2-positive metastatic breast cancer. ${ }^{37}$
Furthermore, the ratio of $\mathrm{Bax} / \mathrm{Bcl} 2$ serves as a marker of cell susceptibility to apoptosis. ${ }^{38}$ A previous clinical study has revealed that a higher level of Bax prolonged the median disease-free survival and increased chemotherapy sensitivity among patients with ovarian cancer. ${ }^{39}$

A notable feature of apoptosis is that it exerts its effects mainly through the activity of a type of serine protease known as caspase, ${ }^{40}$ which cleave and activate each other. The caspase protein family subdivides into sub-families 
A

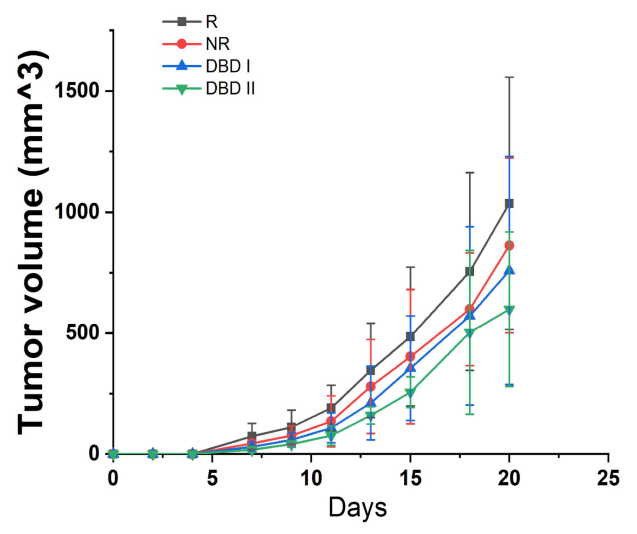

B

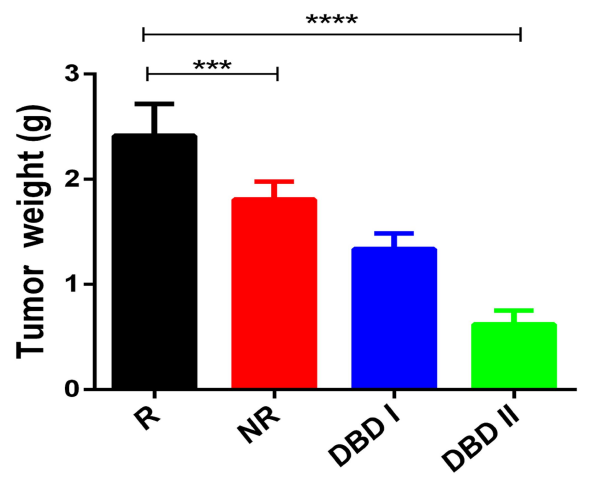

Figure 6 DBD inhibited the growth of metastatic tumor. (A) Growth curve of metastatic tumor. (B) Metastatic tumor weight. All data was presented as mean \pm standard deviation, $* * * p<0.001$, $* * * * p<0.0001$
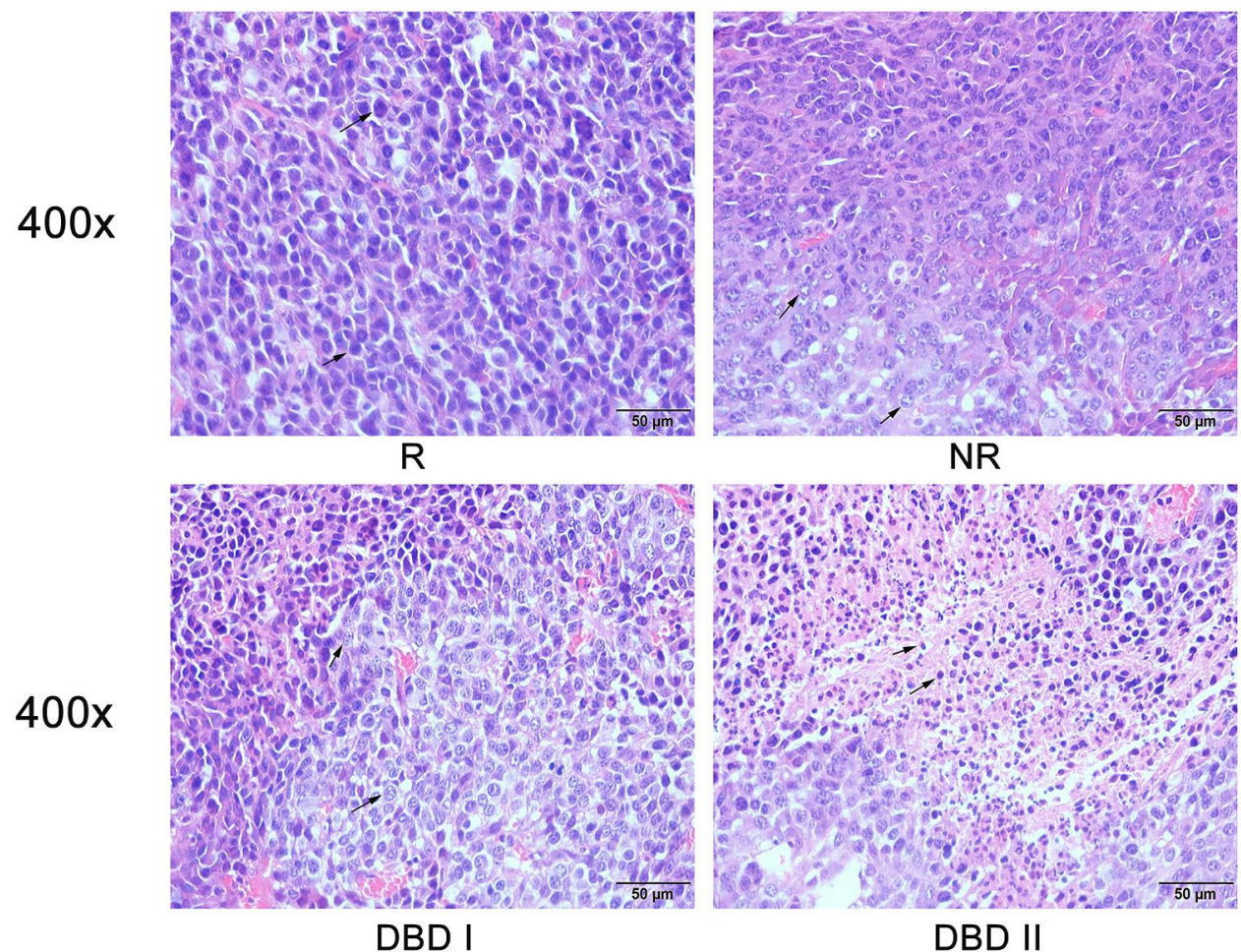

NR

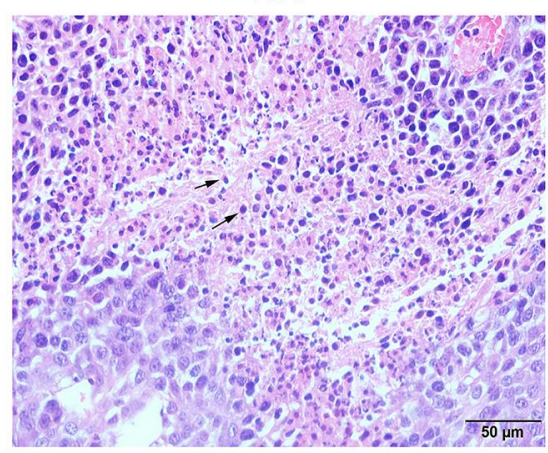

DBD II

Figure 7 Histopathological examination. HE staining of metastatic tumor. Representative image for HE staining of tumor sections from groups R, NR, DBD I and DBD II at 400x magnification power.

based upon function, including initiator (caspases 2, 8, 9, and 10) and effector/executioner (caspases 3, 6, and 7) groups; the latter group exerts apoptotic effects. ${ }^{41}$ Cas 3 is generally regarded as the major apoptotic mediator and a positive marker of an effective therapeutic strategy in cancer. The present findings suggest that DBD upregulates the expression of Bax, Cas3, C-cas3, and increases the ratio of $\mathrm{Bax} / \mathrm{Bcl} 2$, while it down-regulates the expression of $\mathrm{Bcl} 2$; these findings indicate that $\mathrm{DBD}$ may inhibit the progress of MCC by inducing cell apoptosis.

\section{Conclusion}

This study systematically identified the compounds and mechanisms of action involved in DBD in the treatment of MCC using network pharmacology analysis and 
A

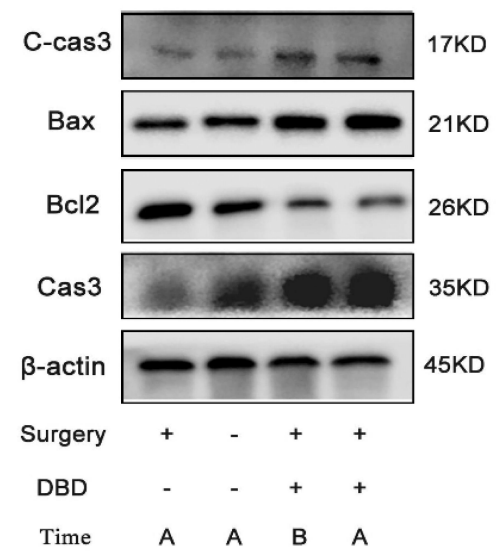

C

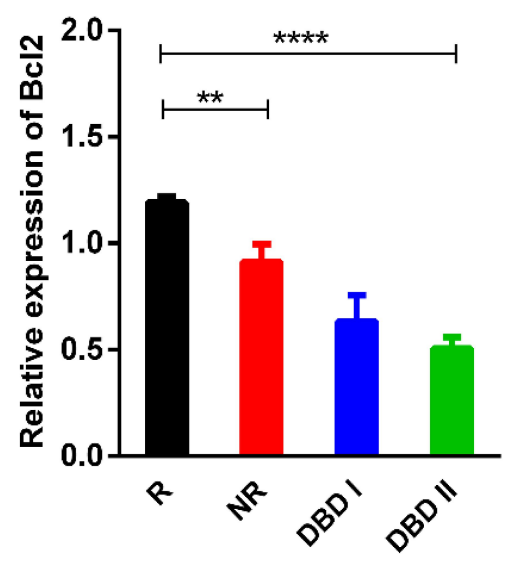

E

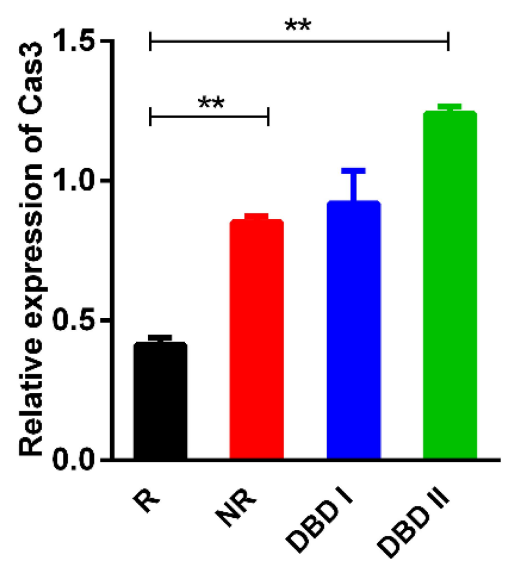

B

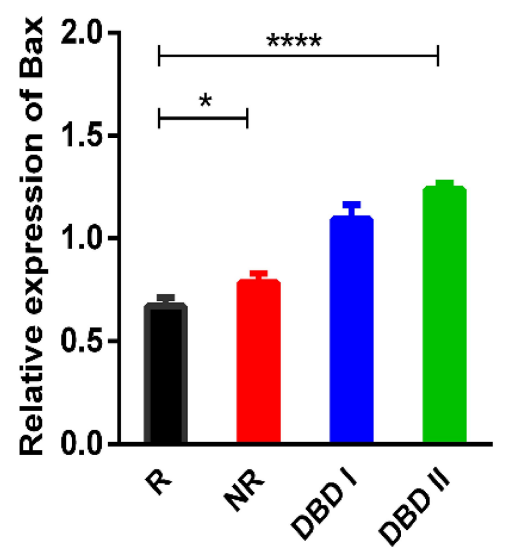

D

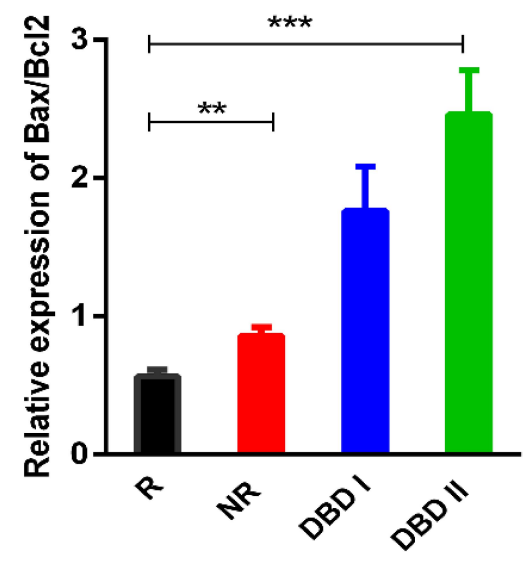

$\mathbf{F}$

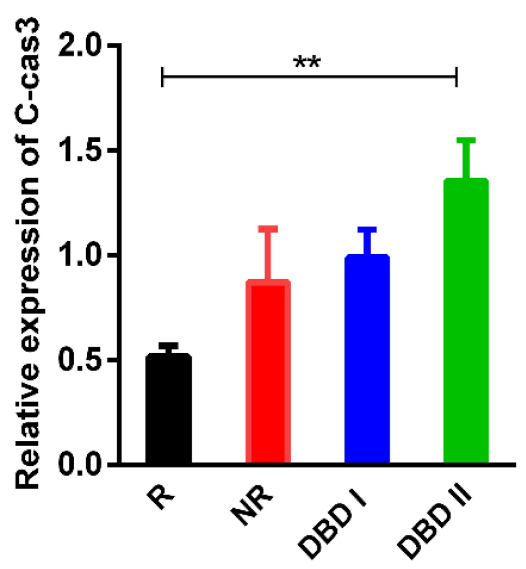

Figure 8 DBD inhibited the growth of metastatic tumor through inducing cell apoptosis. (A) The effect of DBD on metastatic tumor was analyzed by Western blot. Figures (B and $\mathbf{C}, \mathbf{E}$ and $\mathbf{F}$ ) showed the expression of $\mathrm{Bax}, \mathrm{Bcl} 2$, Cas3 and $\mathrm{C}$-cas3 in metastatic tumor among R, NR, DBD I and DBD II groups, with $\beta$-actin as internal control. (D) Western blot analysis showed the ratio of $\mathrm{Bax} / \mathrm{Bcl} 2$ in Metastatic tumor among groups $\mathrm{R}, \mathrm{NR}, \mathrm{DBD}$ I and DBD II. All data was presented as mean \pm standard deviation. $\left({ }^{*} p<0.05,{ }^{*} \mathrm{p}<<0.01,{ }^{*} * *_{\mathrm{p}}<0.001,{ }^{*} * * \mathrm{p}<0.000 \mathrm{I}\right)$.

experimental validation. The present findings support the theory that surgical removal of the primary tumor promotes metastatic tumor growth; In contrast, administration of DBD in the perioperative period could inhibit the progress of MCC after primary tumor resection; its postoperative administration achieved effects superior to those 
achieved by its preoperative administration. This suggests that postoperative application of DBD may achieve better overall effect on metastatic foci in colon cancer patients after primary tumor resection. In addition, the present findings provide some evidence for the application of tonic therapy in tumor treatment at the perioperative period. The limitation of our study is that the candidate compounds of DBD in the treatment of MCC was based on network pharmacology prediction only, more extensive experiments are needed to provide experimental evidence in the future.

\section{Abbreviations}

TCMSP, Traditional Chinese Medicine Systems Pharmacology; OMIM, Online Mendelian Inheritance in Man; GO, Gene Ontology; KEGG, Kyoto Encyclopedia of Genes and Genomes; TCM, Traditional Chinese Medicine; DC, Degree Centrality; BC, Betweenness Centrality; CC, Closeness Centrality.

\section{Acknowledgments}

The authors would like to acknowledge the financial supports from the National Natural Science Foundation of China.

\section{Author Contributions}

All authors made a significant contribution to the work reported, whether that is in the conception, study design, execution, acquisition of data, analysis and interpretation, or in all these areas; took part in drafting, revising or critically reviewing the article; gave final approval of the version to be published; have agreed on the journal to which the article has been submitted; and agree to be accountable for all aspects of the work.

\section{Funding}

This work was supported by the National Natural Science Foundation of China (NSFC) (grant No. 81704033).

\section{Disclosure}

The authors declare that they have no competing interests.

\section{References}

1. Bray F, Ferlay J, Soerjomataram I, Siegel RL, Torre LA, Jemal A. Global cancer statistics 2018: GLOBOCAN estimates of incidence and mortality worldwide for 36 cancers in 185 countries. CA Cancer J Clin. 2018;68(6):394-424. doi:10.3322/caac.21492

2. Demicheli R, Retsky MW, Hrushesky WJ, Baum M, Gukas ID. The effects of surgery on tumor growth: a century of investigations. Ann Oncol. 2008;19(11):1821-1828. doi:10.1093/annonc/mdn386
3. Retsky M, Demicheli R, Hrushesky W, Baum M, Gukas I. Surgery triggers outgrowth of latent distant disease in breast cancer: an inconvenient truth? Cancers. 2010;2(2):305-337. doi:10.3390/ cancers 2020305

4. Horowitz M, Neeman E, Sharon E, Ben-Eliyahu S. Exploiting the critical perioperative period to improve long-term cancer outcomes. Nat Rev Clin Oncol. 2015;12(4):213-226. doi:10.1038/ nrclinonc.2014.224

5. Conrick-Martin I, Kell MR, Buggy DJ. Meta-analysis of the effect of central neuraxial regional anesthesia compared with general anesthesia on postoperative natural killer $\mathrm{T}$ lymphocyte function. $J$ Clin Anesth. 2012;24(1):3-7. doi:10.1016/j.jclinane.2011.09.001

6. Greenfeld K, Avraham R, Benish M, et al. Immune suppression while awaiting surgery and following it: dissociations between plasma cytokine levels, their induced production, and NK cell cytotoxicity. Brain Behav Immun. 2007;21(4):503-513. doi:10.1016/j. bbi.2006.12.006

7. Perez-Sayans M, Somoza-Martin JM, Barros-Angueira F, Diz PG, Gandara Rey JM, Garcia-Garcia A. Beta-adrenergic receptors in cancer: therapeutic implications. Oncol Res. 2010;19(1):45-54. doi:10.3727/096504010x12828372551867

8. Peeters CF, de Waal RM, Wobbes T, Westphal JR, Ruers TJ. Outgrowth of human liver metastases after resection of the primary colorectal tumor: a shift in the balance between apoptosis and proliferation. Int $J$ Cancer. 2006;119(6):1249-1253. doi:10.1002/ ijc. 21928

9. Svendsen MNWK, Nielsen HJ. VEGF and tumour angiogenesis. Impact of surgery, wound healing, inflammation and blood transfusion. Scand J Gastroenterol. 2002;37(4):373-379. doi:10.1080/003655202317315971

10. Zeng XY, Xie H, Yuan J, et al. M2-like tumor-associated macrophages-secreted EGF promotes epithelial ovarian cancer metastasis via activating EGFR-ERK signaling and suppressing lncRNA LIMT expression. Cancer Biol Ther. 2019;20(7):956-966. doi:10.1080/15384047.2018.1564567

11. Yamaguchi KTY, Aoki S. Significant detection of circulating cancer cells in the blood by reverse transcriptase-polymerase chain reaction during colorectal cancer resection. Ann Surg. 2000;232(1):58-65. doi:10.1097/00000658-200007000-00009

12. Gong AG, Li N, Lau KM, et al. Calycosin orchestrates the functions of Danggui Buxue Tang, a Chinese herbal decoction composing of astragali radix and angelica sinensis radix: an evaluation by using calycosin-knock out herbal extract. $J$ Ethnopharmacol. 2015;168:150-157. doi:10.1016/j.jep.2015.03.033

13. Hu G, Yang P, Zeng Y, Zhang S, Song J. Danggui Buxue decoction promotes angiogenesis by up-regulation of VEGFR $1 / 2$ expressions and down-regulation of sVEGFR1/2 expression in myocardial infarction rat. J Chin Med Assoc. 2018;81(1):37-46. doi:10.1016/j. jcma.2017.06.015

14. Zhou J, Li X, Deng P, et al. Chinese herbal formula, modified Danggui Buxue Tang, attenuates apoptosis of hematopoietic stem cells in immune-mediated aplastic anemia mouse model. J Immunol Res. 2017;2017:9786972. doi:10.1155/2017/9786972

15. Li F, Tang R, Chen LB, Zhang KS, Huang XP, Deng CQ. Effects of astragalus combined with angelica on bone marrow hematopoiesis suppression induced by cyclophosphamide in mice. Biol Pharm Bull. 2017;40(5):598-609. doi:10.1248/bpb.b16-00802

16. Cui MD, Pan ZH, Pan LQ. Danggui Buxue extract-loaded liposomes in thermosensitive gel enhance In Vivo Dermal Wound Healing via activation of the VEGF/PI3K/Akt and TGF-beta/Smads signaling pathway. Evid Based Complement Alternat Med. 2017;2017:8407249. doi:10.1155/2017/8407249

17. Gao QT, Cheung JKH, Li J, et al. A Chinese herbal decoction, Danggui Buxue Tang, activates extracellular signal-regulated kinase in cultured T-lymphocytes. FEBS Lett. 2007;581(26):5087-5093. doi:10.1016/j.febslet.2007.09.053 
18. Du QC, Yang KZ, Sun XF. Efficacy of auxiliary therapy with Danggui Buxue Decoction No.1 in treating patients of non-small cell lung cancer at peri-operational stage. Chin J Integr Med. 2009;15(3):184-188. doi:10.1007/s11655-009-0184-y

19. Miao ZSFPY. Effect of Danggui Buxue Decoction on proliferation and its molecular mechanism in tumor vascularvendothelial cells. Chinese J Exp Traditional Med Formulae. 2013;19(2):163-167. doi:10.13422/j.cnki.syfjx.2013.02.055

20. Yue SJ, Xin LT, Fan YC, et al. Herb pair Danggui-Honghua: mechanisms underlying blood stasis syndrome by system pharmacology approach. Sci Rep. 2017;7:40318. doi:10.1038/srep40318

21. Dong TT, Zhao KJ, Gao QT, et al. Chemical and biological assessment of a chinese herbal decoction containing Radix Astragali and Radix Angelicae Sinensis: determination of drug ratio in having optimized properties. J Agric Food Chem. 2006;54(7):2767-2774. doi:10.1021/jf0531631

22. Lin PL, Li ZC, Xie RF, Wang YH, Zhou X. Compatibility study of Danggui Buxue Tang on chemical ingredients, angiogenesis and endothelial function. Sci Rep. 2017;7:45111. doi:10.1038/srep45111

23. B GN F, Saffer EA. Influence of the interval between primary tumor removal and chemotherapy on kinetics and growth of metastases. Cancer Res. 1983;43(4):1488-1492.

24. Gang GJ-H, Han J-H, Liao F, et al. Chinese herbal decoction Shiquan Dabu Tang inhibits tumor growth and angiogenesis of metastasis after primary tumor surgical removal in mice. $J$ Chinese Integrative Med. 2012;10(04):436-447. doi:10.3736/jcim20120413

25. Jin-Fang-Du W-Q-Y, Zhou Y, Sun J, Shi X-L. Effects of Shiquan Dabu Decoction on metastatic tumor growth and NK cells after resection of primary tumor of colon cancer. Shanghai $J$ Traditional Chinese Med. 2017;51(12):79-83.

26. Migliaccio A, Yang F, Song L, et al. Combination of quercetin and 2-methoxyestradiol enhances inhibition of human prostate cancer LNCaP and PC-3 Cells Xenograft tumor growth. PLoS One. 2015;10(5). doi:10.1371/journal.pone.0128277

27. Cheng $\mathrm{L}$, Shi $\mathrm{L}, \mathrm{Wu} \mathrm{J}$, et al. A hederagenin saponin isolated from Clematis ganpiniana induces apoptosis in breast cancer cells via the mitochondrial pathway. Oncol Lett. 2017;15(2):1737-1743. doi:10.3892/ol.2017.7494

28. Woo J-H, Ahn J-H, Jang DS, Lee K-T, Choi J-H. Effect of kumatakenin isolated from cloves on the apoptosis of cancer cells and the alternative activation of tumor-associated macrophages. J Agric Food Chem. 2017;65(36):7893-7899. doi:10.1021/acs.jafc.7b01543

29. Zhang J, Liu L, Wang J, Ren B, Zhang L, Li W. Formononetin, an isoflavone from Astragalus membranaceus inhibits proliferation and metastasis of ovarian cancer cells. $J$ Ethnopharmacol. 2018;221:91-99. doi:10.1016/j.jep.2018.04.014
30. Zhang D, Sun G, Peng L, Tian J, Zhang H. Calycosin inhibits viability, induces apoptosis, and suppresses invasion of cervical cancer cells by upregulating tumor suppressor miR-375. Arch Biochem Biophys. 2020;691:108478. doi:10.1016/j.abb.2020.108478

31. El-Kott AF, Al-Kahtani MA, Shati AA. Calycosin induces apoptosis in adenocarcinoma HT29 cells by inducing cytotoxic autophagy mediated by SIRT1/AMPK-induced inhibition of Akt/mTOR. Clin Exp Pharmacol Physiol. 2019;46(10):944-954. doi:10.1111/14401681.13133

32. Y TC F, Hu HT, et al. Inhibitory effect of trans-ferulic acid on proliferation and migration of human lung cancer cells accompanied with increased endogenous reactive oxygen species and $\beta$-catenin instability. Chin Med. 2016;11(45). doi:10.1186/s13020-016-0116-7

33. Hanahan D, Weinberg RA. Hallmarks of cancer: the next generation. Cell. 2011;144(5):646-674. doi:10.1016/j.cell.2011.02.013

34. Adams JM, Cory S. The Bcl-2 apoptotic switch in cancer development and therapy. Oncogene. 2007;26(9):1324-1337. doi:10.1038/sj. onc. 1210220

35. Campbell KJ, Tait SWG. Targeting BCL-2 regulated apoptosis in cancer. Open Biol. 2018;8(5):May. doi:10.1098/rsob.180002

36. L ZG Y, Li DC. MiR-136 triggers apoptosis in human gastric cancer cells by targeting AEG-1 and BCL2. Eur Rev Med Pharmacol Sci. 2018;22(21):7251-7256. doi:10.26355/eurrev 20181116259

37. Lok SW, Whittle JR, Vaillant F. A phase ib dose-escalation and expansion study of the BCL2 Inhibitor venetoclax combined with tamoxifen in ER and BCL2-Positive metastatic breast cancer. Cancer Discov. 2019;9(3):354-369. doi:10.1158/2159-8290.CD-18-1151

38. Zhang H, Dong R, Zhang P, Wang Y. Songorine suppresses cell growth and metastasis in epithelial ovarian cancer via the $\mathrm{Bcl}-2 /$ Bax and GSK3 $\beta / \beta$-catenin signaling pathways. Oncol Rep. 2019;41 (5):3069-3079. doi:10.3892/or.2019.7070

39. Tai YTLS, Niloff E, Weisman C, Strobel T, Cannistra SA. BAX protein expression and clinical outcome in epithelial ovarian cancer. $J \quad$ Clin Oncol. 1998;16(8):2583-2590. doi:10.1200/ JCO.1998.16.8.2583

40. Xu X, Lai Y, Hua ZC. Apoptosis and apoptotic body: disease message and therapeutic target potentials. Biosci Rep. 2019;39(1). doi:10.1042/BSR20180992

41. Knight T, Luedtke D, Edwards H, Taub JW, Ge Y. A delicate balance - The BCL-2 family and its role in apoptosis, oncogenesis, and cancer therapeutics. Biochem Pharmacol. 2019;162:250-261. doi:10.1016/j. bcp.2019.01.015

\section{Publish your work in this journal}

Drug Design, Development and Therapy is an international, peerreviewed open-access journal that spans the spectrum of drug design and development through to clinical applications. Clinical outcomes, patient safety, and programs for the development and effective, safe, and sustained use of medicines are a feature of the journal, which has also been accepted for indexing on PubMed Central. The manuscript management system is completely online and includes a very quick and fair peer-review system, which is all easy to use. Visit http://www. dovepress.com/testimonials.php to read real quotes from published authors. 